\title{
LINEAR WEINGARTEN HYPERSURFACES IN A REAL SPACE FORM
}

\author{
SHICHANG SHU \\ Department of Mathematics, Xianyang Normal University, Xianyang, Shaanxi 712000, P.R. China \\ e-mail: shushichang@126.com
}

(Received 31 July 2009; accepted 7 April 2010)

\begin{abstract}
In this paper, we investigate linear Weingarten hypersurfaces with two distinct principal curvatures in a real space form $M^{n+1}(c)$, we obtain two rigidity results and give some characterization of the Riemannian product $S^{k}(a) \times S^{n-k}\left(\sqrt{1-a^{2}}\right)$, $1 \leq k \leq n-1$ in $M^{n+1}(c)(c=1)$, the Riemannian product $R^{k} \times S^{n-k}(a), 1 \leq k \leq n-1$ in $M^{n+1}(c)(c=0)$ and the Riemannian product $H^{k}\left(\tanh ^{2} \rho-1\right) \times S^{n-k}\left(\operatorname{coth}^{2} \rho-1\right)$, $1 \leq k \leq n-1$ in $M^{n+1}(c)(c=-1)$.
\end{abstract}

2010 Mathematics Subject Classification. 53C42, 53A10.

1. Introduction. Let $M^{n}$ be an $n$-dimensional hypersurface in a real space form $M^{n+1}(c)$ of dimension $n+1$. It is well known that there are many rigidity results for hypersurfaces in a real space form with constant mean curvature or with constant scalar curvature or with the scalar curvature and the mean curvature being linearly related. For example, one can see [2-6, 8-11].

Recently, H. Li, Y. J. Suh and G. Wei [7] introduced the so called linear Weingarten hypersurface in a unit sphere $S^{n+1}(1)$. We can generalize it to a real space form $M^{n+1}(c)$, that is, a hypersurface in a real space form $M^{n+1}(c)$ is called a linear Weingarten hypersurface if the scalar curvature $R$ and the mean curvature $H$ satisfy the linear relation $\alpha R+\beta H+\gamma=0$, where $\alpha, \beta$ and $\gamma$ are constants such that $\alpha^{2}+\beta^{2} \neq 0$.

We easily see that if the constant $\alpha=0$, a linear Weingarten hypersurface reduces to a hypersurface with constant mean curvature. If the constant $\beta=0$, a linear Weingarten hypersurface reduces to a hypersurface with constant scalar curvature. If the constant $\gamma=0$, a linear Weingarten hypersurface reduces to a hypersurface with the scalar curvature and the mean curvature being linearly related, which was studied by $\mathrm{H}$. Li [6] for the unit sphere. Therefore, we know that the linear Weingarten hypersurface is a natural generalization of hypersurface with constant mean curvature or with constant scalar curvature or the scalar curvature and the mean curvature being linearly related.

In this paper, we try to study the linear Weingarten hypersurfaces with two distinct principal curvatures in a real space form $M^{n+1}(c)$. In order to state our theorem clearly, we introduce the well-known standard models of complete hypersurfaces in $M^{n+1}(c)$. Let $N_{k, n-k}:=R^{k} \times S^{n-k}(a)$. Then $N_{k, n-k}$ has two distinct constant principal curvatures 0 and $\sqrt{a}$ with multiplicities $k$ and $n-k$, respectively. Let $M_{k, n-k}:=S^{k}(a) \times$ $S^{n-k}\left(\sqrt{1-a^{2}}\right)$. Then $M_{k, n-k}$ has two distinct constant principal curvatures

$$
\lambda_{1}=\cdots=\lambda_{k}=\frac{\sqrt{1-a^{2}}}{a}, \quad \lambda_{k+1}=\cdots=\lambda_{n}=-\frac{a}{\sqrt{1-a^{2}}} .
$$


Let $T_{k, \rho}:=\left\{x \in H^{n+1}(-1) \mid-x_{0}^{2}+x_{1}^{2}+\cdots+x_{k}^{2}=-\cosh ^{2} \rho\right\}, \rho>0,1 \leq k \leq n-1$. Then $T_{k, \rho}$ has two distinct constant principal curvatures

$$
\lambda_{1}=\cdots=\lambda_{k}=\tanh \rho, \quad \lambda_{k+1}=\cdots=\lambda_{n}=\operatorname{coth} \rho .
$$

Moreover, $T_{k, \rho}$ is isometric to the Riemannian product $H^{k}\left(\tanh ^{2} \rho-1\right) \times$ $S^{n-k}\left(\operatorname{coth}^{2} \rho-1\right)$. We shall prove the following:

MAIN THEOREM 1.1. Let $M^{n}$ be an $n(n \geq 3)$-dimensional complete connected and oriented linear Weingarten hypersurface in a real space form $M^{n+1}(c)$ with two distinct principal curvatures. Then

(1) if the multiplicities of both principal curvatures are greater than 1 , then

(i) for $c=1, M^{n}$ is isometric to a Riemannian product $S^{k}(a) \times S^{n-k}\left(\sqrt{1-a^{2}}\right)$, where $1<k<n-1$;

(ii) for $c=0, M^{n}$ is isometric to a Riemannian product $R^{k} \times S^{n-k}(a)$, where $1<$ $k<n-1$;

(iii) for $c=-1, M^{n}$ is isometric to a Riemannian product $H^{k}\left(\tanh ^{2} \rho-1\right) \times$ $S^{n-k}\left(\operatorname{coth}^{2} \rho-1\right)$, where $1<k<n-1$.

(2) if $M^{n}$ has two distinct principal curvatures $\lambda$ and $\mu$ of multiplicities $n-1$ and 1 , assume that the sectional curvature of $M^{n}$ is non-negative and $\lambda \neq-\frac{\beta}{2 \alpha n(n-1)}$, $\gamma \alpha+\alpha^{2} n(n-1) c=\frac{\beta^{2}}{4 n(n-1)}$, then

(i) for $c=1, M^{n}$ is isometric to a Riemannian product $S^{1}(a) \times S^{n-1}\left(\sqrt{1-a^{2}}\right)$;

(ii) for $c=0$ and $\beta \neq 0, M^{n}$ is isometric to a Riemannian product $R^{1} \times S^{n-1}(a)$ or $R^{n-1} \times S^{1}(a)$

(iii) for $c=-1$ and $\beta^{2}-4 \alpha^{2} n^{2}(n-1)^{2}>0, M^{n}$ is isometric to a Riemannian product $H^{1}\left(\tanh ^{2} \rho-1\right) \times S^{n-1}\left(\operatorname{coth}^{2} \rho-1\right)$ or $H^{n-1}\left(\tanh ^{2} \rho-1\right) \times S^{1}\left(\operatorname{coth}^{2} \rho-1\right)$.

Denote by $P(t)$ and $S(t)$ the following functions:

$$
P(t)=c-\frac{\beta}{4 \alpha(n-1)} t-\frac{n-2}{2} t^{2},
$$

and

$$
S(t)=\frac{n^{2}}{4} t^{2}+\frac{(n-2) \beta}{4 \alpha(n-1)} t+\frac{\beta^{2}}{16 \alpha^{2}(n-1)^{2}} .
$$

From Lemma 3.3, we know that $P(t)$ has two distinct real roots $t_{1}, t_{2}$. From (3.29), we know that $S(t)$ is the squared norm of the second fundamental form of $M^{n}$. We can prove the following:

MAIN THEOREM 1.2. Let $M^{n}$ be an $n(n \geq 3)$-dimensional complete connected and oriented linear Weingarten hypersurface in a real space form $M^{n+1}(c)$ with two distinct principal curvatures $\lambda$ and $\mu$ of multiplicities $n-1$ and 1 . If $\lambda \neq-\frac{\beta}{2 \alpha n(n-1)}$, $\gamma \alpha+\alpha^{2} n(n-1) c=\frac{\beta^{2}}{4 n(n-1)}$ and the squared norm of the second fundamental form of $M^{n}$ satisfies one of the following conditions

(1) $\min \left(S\left(t_{1}\right), S\left(t_{2}\right)\right) \leq S \leq \max \left(S\left(t_{1}\right), S\left(t_{2}\right)\right)$ or

(2) $S \geq \max \left(S\left(t_{1}\right), S\left(t_{2}\right)\right)$ or

(3) $S \leq \min \left(S\left(t_{1}\right), S\left(t_{2}\right)\right)$, then

(i) for $c=1, M^{n}$ is isometric to a Riemannian product $S^{1}(a) \times S^{n-1}\left(\sqrt{1-a^{2}}\right)$; 
(ii) for $c=0$ and $\beta \neq 0, M^{n}$ is isometric to a Riemannian product $R^{1} \times S^{n-1}(a)$ or $R^{n-1} \times S^{1}(a)$

(iii) for $c=-1$ and $\beta^{2}-4 \alpha^{2} n^{2}(n-1)^{2}>0, M^{n}$ is isometric to a Riemannian product $H^{1}\left(\tanh ^{2} \rho-1\right) \times S^{n-1}\left(\operatorname{coth}^{2} \rho-1\right)$ or $H^{n-1}\left(\tanh ^{2} \rho-1\right) \times S^{1}\left(\operatorname{coth}^{2} \rho-1\right)$, where $t_{1}, t_{2}$ are the two distinct real roots of (1.1) and $S(t)$ is denoted by (1.2).

REMARK 1.3. If $\alpha=0$, that is, $M^{n}$ is a hypersurface with constant mean curvature, the result of (1) in Main Theorem 1.1 reduces to the result of G. Wei [11] for $c=1$. If $\beta=0$, that is, $M^{n}$ is a hypersurface with constant scalar curvature, the results of (1) in Main Theorem 1.1 reduce to the results of Cheng $[3,4]$ for $c=1, c=0$ and Z. Hu et al. [5] for $c=-1$, respectively. We should notice that our Main Theorems also generalize some important results of [3-5] and of authors [10], in which the hypersurfaces with constant mean curvature or with constant scalar curvature were investigated, to linear Weingarten hypersurface in a real space form $M^{n+1}(c)$.

2. Preliminaries. Let $M^{n+1}(c)$ be an $(n+1)$-dimensional connected Riemannian manifold with constant sectional curvature $c$. Let $M^{n}$ be an $n$-dimensional hypersurface in $M^{n+1}(c)$. We choose a local orthonormal frame $e_{1}, \ldots, e_{n+1}$ in $M^{n+1}(c)$ such that $e_{1}, \ldots, e_{n}$ are tangent to $M^{n}$. Let $\omega_{1}, \ldots, \omega_{n+1}$ be the dual coframe. We use the following convention on the range of indices:

$$
1 \leq A, B, C, \ldots \leq n+1 ; 1 \leq i, j, k, \ldots \leq n .
$$

The structure equations of $M^{n+1}(c)$ are given by

$$
\begin{gathered}
d \omega_{A}=\sum_{B} \omega_{A B} \wedge \omega_{B}, \quad \omega_{A B}+\omega_{B A}=0 \\
d \omega_{A B}=\sum_{C} \omega_{A C} \wedge \omega_{C B}-\frac{1}{2} \sum_{C, D} K_{A B C D} \omega_{C} \wedge \omega_{D}, \\
K_{A B C D}=c\left(\delta_{A C} \delta_{B D}-\delta_{A D} \delta_{B C}\right) .
\end{gathered}
$$

Restricting to $M^{n}$,

$$
\begin{gathered}
\omega_{n+1}=0 . \\
\omega_{n+1 i}=\sum_{j} h_{i j} \omega_{j}, \quad h_{i j}=h_{j i} .
\end{gathered}
$$

The structure equations of $M^{n}$ are

$$
\begin{gathered}
d \omega_{i}=\sum_{j} \omega_{i j} \wedge \omega_{j}, \quad \omega_{i j}+\omega_{j i}=0, \\
d \omega_{i j}=\sum_{k} \omega_{i k} \wedge \omega_{k j}-\frac{1}{2} \sum_{k, l} R_{i j k l} \omega_{k} \wedge \omega_{l}, \\
R_{i j k l}=c\left(\delta_{i k} \delta_{j l}-\delta_{i l} \delta_{j k}\right)+\left(h_{i k} h_{j l}-h_{i l} h_{j k}\right), \\
n(n-1)(r-c)=n^{2} H^{2}-S,
\end{gathered}
$$


where $n(n-1) r=R$ is the scalar curvature, $H$ is the mean curvature and $S$ is the squared norm of the second fundamental form of $M$.

We choose $e_{1}, \ldots, e_{n}$ such that $h_{i j}=\lambda_{i} \delta_{i j}$. From (2.5) we have

$$
\omega_{n+1 i}=\lambda_{i} \omega_{i}, \quad i=1,2, \ldots, n .
$$

Hence, we have from the structure equations of $M^{n}$

$$
\begin{aligned}
d \omega_{n+1 i} & =d \lambda_{i} \wedge \omega_{i}+\lambda_{i} d \omega_{i} \\
& =d \lambda_{i} \wedge \omega_{i}+\lambda_{i} \sum_{j} \omega_{i j} \wedge \omega_{j} .
\end{aligned}
$$

On the other hand, we have on the curvature forms of $M^{n+1}(c)$,

$$
\begin{aligned}
\Omega_{n+1 i} & =-\frac{1}{2} \sum_{C, D} K_{n+1 i C D} \omega_{C} \wedge \omega_{D} \\
& =-\frac{1}{2} \sum_{C, D} c\left(\delta_{n+1 C} \delta_{i D}-\delta_{n+1 D} \delta_{i C}\right) \omega_{C} \wedge \omega_{D} \\
& =-c \omega_{n+1} \wedge \omega_{i}=0 .
\end{aligned}
$$

Therefore, from the structure equations of $M^{n+1}(c)$, we have

$$
\begin{aligned}
d \omega_{n+1 i} & =\sum_{j} \omega_{n+1 j} \wedge \omega_{j i}+\omega_{n+1 n+1} \wedge \omega_{n+1 i}+\Omega_{n+1 i} \\
& =\sum_{j} \lambda_{j} \omega_{i j} \wedge \omega_{j} .
\end{aligned}
$$

From (2.11) and (2.13), we obtain

$$
d \lambda_{i} \wedge \omega_{i}+\sum_{j}\left(\lambda_{i}-\lambda_{j}\right) \omega_{i j} \wedge \omega_{j}=0
$$

and can write

$$
\psi_{i j}=\left(\lambda_{i}-\lambda_{j}\right) \omega_{i j}
$$

As $\psi_{i j}=\psi_{j i},(2.14)$ can be written as

$$
\sum_{j}\left(\psi_{i j}+\delta_{i j} d \lambda_{j}\right) \wedge \omega_{j}=0
$$

By E. Cartan's Lemma, we get

$$
\psi_{i j}+\delta_{i j} d \lambda_{j}=\sum_{k} Q_{i j k} \omega_{k},
$$

where $Q_{i j k}$ are uniquely determined functions such that

$$
Q_{i j k}=Q_{i k j}
$$


3. Proof of main Theorems. We firstly have the following Proposition 3.1 original due to Otsuki [8].

Proposition 3.1 ([8]). Let $M^{n}$ be a hypersurface in a real space form $M^{n+1}(c)$ such that the multiplicities of the principal curvatures are constant. Then the distribution of the space of the principal vectors corresponding to each principal curvature is completely integrable. In particular, if the multiplicity of a principal curvature is greater than 1 , then this principal curvature is constant on each integral submanifold of the corresponding distribution of the space of the principal vectors.

Proof of (1) in Main Theorem 1.1. Let $\lambda, \mu$ be the principal curvatures of multiplicities $k$ and $n-k$ respectively, where $1<k<n-1$. By (2.9) and $\alpha R+\beta H+$ $\gamma=0$, we have

$$
\begin{aligned}
& \alpha n k(k-1) \lambda^{2}+2 \alpha n k(n-k) \lambda \mu+\alpha n(n-k)(n-k-1) \mu^{2} \\
& \quad+\beta k \lambda+\beta(n-k) \mu+\gamma n+\alpha n^{2}(n-1) c=0 .
\end{aligned}
$$

Denote by $D_{\lambda}$ and $D_{\mu}$ the integral submanifolds of the corresponding distribution of the space of principal vectors corresponding to the principal curvature $\lambda$ and $\mu$, respectively. From Proposition 3.1, we know that $\lambda$ is constant on $D_{\lambda}$. From (3.1), we infer that $\mu$ is constant on $D_{\lambda}$. By making use of Proposition 3.1 again, we have $\mu$ is constant on $D_{\mu}$. Therefore, we know that $\mu$ is constant on $M^{n}$. By the same assertion we know that $\lambda$ is constant on $M^{n}$. Therefore $M^{n}$ is isoparametric. By E. Cartan [1], we know that $M^{n}$ is isometric to the Riemannin product $R^{k} \times S^{n-k}(a)$ for $c=0$, or $S^{k}(a) \times S^{n-k}\left(\sqrt{1-a^{2}}\right)$ for $c=1$, or $H^{k}\left(\tanh ^{2} \rho-1\right) \times S^{n-k}\left(\operatorname{coth}^{2} \rho-\right.$ 1) for $c=-1$, where $1<k<n-1$. This completes the proof of (1) in Main Theorem 1.1.

REMARK. In fact, we note that Theorem 1.1 is right for general Weingarten hypersurfaces satisfying a differentiable function relating the mean curvature and the scalar curvature of $M^{n}$, i.e. a Weingarten relation $\mathcal{W}(R ; H)=0$.

Let $M^{n}$ be an $n$-dimensional complete linear Weingarten hypersurface with two distinct principal curvatures one of which is simple, that is, without loss of generality, we may assume

$$
\lambda_{1}=\lambda_{2}=\cdots=\lambda_{n-1}=\lambda, \quad \lambda_{n}=\mu,
$$

where $\lambda_{i}$ for $i=1,2, \ldots, n$ are the principal curvatures of $M^{n}$. From (2.9) and $\alpha R+$ $\beta H+\gamma=0$, we obtain that

$$
\alpha n(n-1)(n-2) \lambda^{2}+[2 \alpha n(n-1) \lambda+\beta] \mu+\beta(n-1) \lambda+\gamma n+\alpha n^{2}(n-1) c=0 .
$$

Since we assume that $\lambda \neq-\frac{\beta}{2 \alpha n(n-1)}$ and $\gamma \alpha+\alpha^{2} n(n-1) c=\frac{\beta^{2}}{4 n(n-1)}$. By a direct calculation, (3.2) can be written as

$$
[2 \alpha n(n-1) \lambda+\beta]\left[\mu+\frac{2 \alpha(n-1)(n-2) \lambda+\beta}{4 \alpha(n-1)}\right]=0 .
$$


Thus, we have

$$
\mu=-\frac{2 \alpha(n-1)(n-2) \lambda+\beta}{4 \alpha(n-1)},
$$

and

$$
\lambda-\mu=n \frac{2 \alpha n(n-1) \lambda+\beta}{4 \alpha n(n-1)} .
$$

Let $\varpi=\left|[2 \alpha n(n-1) \lambda+\beta]^{2}\right|^{-\frac{1}{n}}$. We denote the integral submanifold through $x \in M^{n}$ corresponding to $\lambda$ by $M_{1}^{n-1}(x)$. Putting

$$
d \lambda=\sum_{k=1}^{n} \lambda,{ }_{k} \omega_{k}, \quad d \mu=\sum_{k=1}^{n} \mu, \omega_{k} \omega_{k}
$$

From Proposition 3.1, we have

$$
\lambda,_{1}=\lambda,_{2}=\cdots=\lambda,_{n-1}=0 \text { on } M_{1}^{n-1}(x) .
$$

From (3.4), we have

$$
d \mu=-\frac{n-2}{2} d \lambda
$$

Hence, we also have

$$
\mu,,_{1}=\mu, 2=\cdots=\mu,_{n-1}=0 \text { on } M_{1}^{n-1}(x) .
$$

In this case, we may consider locally $\lambda$ is a function of the arc length $s$ of the integral curve of the principal vector field $e_{n}$ corresponding to the principal curvature $\mu$. From (2.17) and (3.7), we have for $1 \leq j \leq n-1$,

$$
\begin{aligned}
d \lambda & =d \lambda_{j}=\sum_{k=1}^{n} Q_{j j k} \omega_{k} \\
& =\sum_{k=1}^{n-1} Q_{j j k} \omega_{k}+Q_{j j n} \omega_{n}=\lambda,{ }_{n} \omega_{n} .
\end{aligned}
$$

Therefore, we have

$$
Q_{i j k}=0, \quad 1 \leq k \leq n-1, \text { and } Q_{j j n}=\lambda,,_{n} .
$$

By (2.17) and (3.9), we have

$$
\begin{aligned}
d \mu & =d \lambda_{n}=\sum_{k=1}^{n} Q_{n n k} \omega_{k} \\
& =\sum_{k=1}^{n-1} Q_{n n k} \omega_{k}+Q_{n n n} \omega_{n}=\sum_{i=1}^{n} \mu,{ }_{i} \omega_{i}=\mu,{ }_{n} \omega_{n} .
\end{aligned}
$$


Hence, we obtain

$$
Q_{n n k}=0, \quad 1 \leq k \leq n-1, \text { and } Q_{n n n}=\mu,{ }_{n} .
$$

From (3.8), we get

$$
Q_{n n n}=\mu,_{n}=-\frac{n-2}{2} \lambda,_{n} .
$$

From the definition of $\psi_{i j}$, if $i \neq j$, we have $\psi_{i j}=0$ for $1 \leq i \leq n-1$ and $1 \leq j \leq n-1$. Therefore, from (2.17), if $i \neq j$ and $1 \leq i \leq n-1$ and $1 \leq j \leq n-1$ we have

$$
Q_{i j k}=0 \text {, for any } k \text {. }
$$

By (2.17), (3.11), (3.13), (3.14) and (3.15), we get

$$
\begin{aligned}
\psi_{j n} & =\sum_{k=1}^{n} Q_{j n k} \omega_{k} \\
& =Q_{j j n} \omega_{j}+Q_{j n n} \omega_{n}=\lambda,{ }_{n} \omega_{j} .
\end{aligned}
$$

From (2.15), (3.5) and (3.16) we have

$$
\begin{aligned}
\omega_{j n} & =\frac{\psi_{j n}}{\lambda-\mu}=\frac{\lambda,,_{n}}{\lambda-\mu} \omega_{j} \\
& =\frac{\lambda, n}{n \frac{2 \alpha n(n-1) \lambda+\beta}{4 \alpha n(n-1)}} \omega_{j} \\
& =\frac{4 \alpha n(n-1) \lambda, n}{n[2 \alpha n(n-1) \lambda+\beta]} \omega_{j} .
\end{aligned}
$$

Thus, from the structure equations of $M^{n}$ we have

$$
d \omega_{n}=\sum_{k=1}^{n-1} \omega_{k} \wedge \omega_{k n}+\omega_{n n} \wedge \omega_{n}=0 .
$$

Therefore, we may put $\omega_{n}=d s$. By (3.10) and (3.12), we get

$$
d \lambda=\lambda,_{n} d s, \quad \lambda,{ }_{n}=\frac{d \lambda}{d s}
$$

and

$$
d \mu=\mu,_{n} d s, \quad \mu,,_{n}=\frac{d \mu}{d s} .
$$

Then we have

$$
\begin{aligned}
\omega_{j n} & =\frac{4 \alpha n(n-1) \lambda, n}{n[2 \alpha n(n-1) \lambda+\beta]} \omega_{j} \\
& =\frac{4 \alpha n(n-1) \frac{d \lambda}{d s}}{n[2 \alpha n(n-1) \lambda+\beta]} \omega_{j} \\
& =\frac{d\left\{\log \left|[2 \alpha n(n-1) \lambda+\beta]^{2}\right|^{\frac{1}{n}}\right\}}{d s} \omega_{j} .
\end{aligned}
$$


From (3.18) and the structure equations of $M^{n+1}(c)$, we have

$$
\begin{aligned}
d \omega_{j n} & =\sum_{k=1}^{n-1} \omega_{j k} \wedge \omega_{k n}+\omega_{j n} \wedge \omega_{n n}+\omega_{j n+1} \wedge \omega_{n+1 n}+\Omega_{j n} \\
& =\sum_{k=1}^{n-1} \omega_{j k} \wedge \omega_{k n}+\omega_{j n+1} \wedge \omega_{n+1 n}-c \omega_{j} \wedge \omega_{n} \\
& =\frac{d\left\{\log \left|[2 \alpha n(n-1) \lambda+\beta]^{2}\right|^{\frac{1}{n}}\right\}}{d s} \sum_{k=1}^{n-1} \omega_{j k} \wedge \omega_{k}-(\lambda \mu+c) \omega_{j} \wedge d s
\end{aligned}
$$

From (3.18), we have

$$
\begin{aligned}
d \omega_{j n}= & \frac{d^{2}\left\{\log \left|[2 \alpha n(n-1) \lambda+\beta]^{2}\right|^{\frac{1}{n}}\right\}}{d s^{2}} d s \wedge \omega_{j} \\
& +\frac{d\left\{\log \left|[2 \alpha n(n-1) \lambda+\beta]^{2}\right|^{\frac{1}{n}}\right\}}{d s} d \omega_{j} \\
= & \frac{d^{2}\left\{\log \left|[2 \alpha n(n-1) \lambda+\beta]^{2}\right|^{\frac{1}{n}}\right\}}{d s^{2}} d s \wedge \omega_{j} \\
& +\frac{d\left\{\log \left|[2 \alpha n(n-1) \lambda+\beta]^{2}\right|^{\frac{1}{n}}\right\}}{d s} \sum_{k=1}^{n} \omega_{j k} \wedge \omega_{k} \\
= & \left\{-\frac{d^{2}\left\{\log \left|[2 \alpha n(n-1) \lambda+\beta]^{2}\right|^{\frac{1}{n}}\right\}}{d s^{2}}\right. \\
& \left.+\left[\frac{d\left\{\log \left|[2 \alpha n(n-1) \lambda+\beta]^{2}\right|^{\frac{1}{n}}\right\}}{d s}\right]^{2}\right\} \omega_{j} \wedge d s \\
& +\frac{d\left\{\log \left|[2 \alpha n(n-1) \lambda+\beta]^{2}\right|^{\frac{1}{n}}\right\}}{d s} \sum_{k=1}^{n-1} \omega_{j k} \wedge \omega_{k} .
\end{aligned}
$$

From the above two equalities, we have

$$
\frac{d^{2}\left\{\log \left|[2 \alpha n(n-1) \lambda+\beta]^{2}\right|^{\frac{1}{n}}\right\}}{d s^{2}}-\left\{\frac{d\left\{\log \left|[2 \alpha n(n-1) \lambda+\beta]^{2}\right|^{\frac{1}{n}}\right\}}{d s}\right\}^{2}-(\lambda \mu+c)=0
$$

Since we define $\varpi=\left|[2 \alpha n(n-1) \lambda+\beta]^{2}\right|^{-\frac{1}{n}}$, we obtain from the above equation

$$
\frac{d^{2} \varpi}{d s^{2}}+\varpi(\lambda \mu+c)=0
$$

We can prove the following Lemma:

LEMMA 3.2. The positive function $\varpi$ is bounded if $c>0$; or $c=0$ and $\beta \neq 0$; or $c<0$ and $\beta^{2}+4 c \alpha^{2} n^{2}(n-1)^{2}>0$. 
Proof. From (3.4) and (3.20), we get

$$
\frac{d^{2} \varpi}{d s^{2}}+\varpi\left[c-\frac{\beta}{4 \alpha(n-1)} \lambda-\frac{n-2}{2} \lambda^{2}\right]=0 .
$$

Since $\varpi=\left|[2 \alpha n(n-1) \lambda+\beta]^{2}\right|^{-\frac{1}{n}}$, we have

$$
\lambda=\frac{ \pm \varpi^{-\frac{n}{2}}-\beta}{2 \alpha n(n-1)} .
$$

Thus, we have from (3.21) that

$$
\frac{d^{2} \varpi}{d s^{2}}+\varpi\left[c-\frac{\beta\left( \pm \varpi^{-\frac{n}{2}}-\beta\right)}{8 \alpha^{2} n(n-1)^{2}}-\frac{(n-2)\left( \pm \varpi^{-\frac{n}{2}}-\beta\right)^{2}}{8 \alpha^{2} n^{2}(n-1)^{2}}\right]=0 .
$$

By making use of the following integral formula

$$
\int u^{m}\left(a+b u^{q}\right)^{p} d u=\frac{u^{m+1}\left(a+b u^{q}\right)^{p}}{p q+m+1}+\frac{a p q}{p q+m+1} \int u^{m}\left(a+b u^{q}\right)^{p-1} d u,
$$

where all $m, p, q, a, b$ are not zero and all $m, p, q$ are rational number, we have

$$
\begin{aligned}
& -\int \frac{(n-2) \varpi\left( \pm \varpi^{-\frac{n}{2}}-\beta\right)^{2}}{8 \alpha^{2} n^{2}(n-1)^{2}} d \varpi \\
& =\frac{\varpi^{2}\left( \pm \varpi^{-\frac{n}{2}}-\beta\right)^{2}}{8 \alpha^{2} n^{2}(n-1)^{2}}+\int \frac{\beta \varpi\left( \pm \varpi^{-\frac{n}{2}}-\beta\right)}{8 \alpha^{2} n(n-1)^{2}} d \varpi .
\end{aligned}
$$

Integrating (3.22) and from (3.23), we have

$$
\left(\frac{d \varpi}{d s}\right)^{2}+\varpi^{2}\left[c+\frac{\left( \pm \varpi^{-\frac{n}{2}}-\beta\right)^{2}}{4 \alpha^{2} n^{2}(n-1)^{2}}\right]=C,
$$

where $C$ is a constant. Thus, we have

$$
\varpi^{2}\left[c+\frac{\left( \pm \varpi^{-\frac{n}{2}}-\beta\right)^{2}}{4 \alpha^{2} n^{2}(n-1)^{2}}\right] \leq C .
$$

If the positive function $\varpi$ is not bounded, that is, $\lim _{s \rightarrow+\infty} \varpi(s)=+\infty$. From (3.25), we have

$$
+\infty\left(c+\frac{\beta^{2}}{4 \alpha^{2} n^{2}(n-1)^{2}}\right) \leq C
$$

Since $c+\frac{\beta^{2}}{4 \alpha^{2} n^{2}(n-1)^{2}}>0$ if $c>0$; or $c=0$ and $\beta \neq 0$; or $c<0$ and $\beta^{2}+4 c \alpha^{2} n^{2}(n-$ $1)^{2}>0$. We have a contradiction from (3.26). This completes the proof of Lemma 3.2.

Proof of (2) in Main Theorem 1.1. If the sectional curvature of $M^{n}$ is non-negative, that is, for $i \neq j, R_{i j i j}=c+\lambda_{i} \lambda_{j} \geq 0$, we have $c+\lambda \mu \geq 0$. From (3.20), we have $\frac{d^{2} w}{d s^{2}} \leq 0$. Thus, $\frac{d \sigma}{d s}$ is a monotonic function of $s \in(-\infty,+\infty)$. Therefore, by the similar assertion in Wei [11], we have $\varpi(s)$ must be monotonic when $s$ tends to infinity. From Lemma 3.2, 
we know that the positive function $\varpi(s)$ is bounded. Since $\varpi(s)$ is bounded and monotonic when $s$ tends to infinity, we know that both $\lim _{s \rightarrow-\infty} \varpi(s)$ and $\lim _{s \rightarrow+\infty} \varpi(s)$ exist and then we get

$$
\lim _{s \rightarrow-\infty} \frac{d \varpi(s)}{d s}=\lim _{s \rightarrow+\infty} \frac{d \varpi(s)}{d s}=0 .
$$

From the monotonicity of $\frac{d \varpi(s)}{d s}$, we have $\frac{d \varpi(s)}{d s} \equiv 0$ and $\varpi(s)=$ constant. From $\varpi=\left|[2 \alpha n(n-1) \lambda+\beta]^{2}\right|^{-\frac{1}{n}}$ and (3.4), we have $\lambda$ and $\mu$ are constant, that is, $M^{n}$ is isoparametric. According to Cartan [1], we know that $M^{n}$ is isometric to the Riemannin product $R^{1} \times S^{n-1}(a)$ or $R^{n-1} \times S^{1}(a)$ for $c=0$ and $\beta \neq 0$; or $S^{1}(a) \times$ $S^{n-1}\left(\sqrt{1-a^{2}}\right)$ for $c=1$; or $H^{1}\left(\tanh ^{2} \rho-1\right) \times S^{n-1}\left(\operatorname{coth}^{2} \rho-1\right)$ or $H^{n-1}\left(\tanh ^{2} \rho-\right.$ 1) $\times S^{1}\left(\operatorname{coth}^{2} \rho-1\right)$ for $c=-1$ and $\beta^{2}-4 \alpha^{2} n^{2}(n-1)^{2}>0$. This completes the proof of (2) in Main Theorem 1.1.

We can also prove the following Lemmas:

LEMMA 3.3. Let

$$
P(t)=c-\frac{\beta}{4 \alpha(n-1)} t-\frac{n-2}{2} t^{2},
$$

and $t^{\prime}=-\frac{\beta}{4 \alpha(n-1)(n-2)}$. If $c+\frac{\beta^{2}}{4 \alpha^{2} n^{2}(n-1)^{2}}>0$, then $P(t)$ has two distinct real roots $t_{1}, t_{2}$ and

(i) if $t \geq t^{\prime}$, then $t \geq t_{2}$ holds if and only if $P(t) \leq 0$ and $t \leq t_{2}$ holds if and only if $P(t) \geq 0$.

(ii) if $t \leq t^{\prime}$, then $t \leq t_{1}$ holds if and only if $P(t) \leq 0$ and $t \geq t_{1}$ holds if and only if $P(t) \geq 0$.

Proof. We have

$$
\frac{d P(t)}{d t}=-\frac{\beta}{4 \alpha(n-1)}-(n-2) t .
$$

it follows that the solution of $\frac{d P_{H}(t)}{d t}=0$ is $t^{\prime}=-\frac{\beta}{4 \alpha(n-1)(n-2)}$. Therefore, we know that if $t \leq t^{\prime}$ if and only if $P(t)$ is an increasing function, $t \geq t^{\prime}$ if and only if $P(t)$ is a decreasing function and $P(t)$ obtains its maximum at $t=t^{\prime}$.

Since $P(t)$ is continuous and $c+\frac{\beta^{2}}{4 \alpha^{2} n^{2}(n-1)^{2}}>0$, we have $P\left(t^{\prime}\right)=c+$ $\frac{\beta^{2}}{32 \alpha^{2}(n-1)^{2}(n-2)}>0$. Therefore, we know that $P(t)$ has two distinct real roots $t_{1}, t_{2}$ and $t_{1}<t^{\prime}<t_{2}$.

(i) If $t \geq t^{\prime}$, from the decreasing property of $P(t)$, we obtain that $t \geq t_{2}$ holds if and only if $P(t) \leq P\left(t_{2}\right)=0$ and $t \leq t_{2}$ holds if and only if $P(t) \geq P\left(t_{2}\right)=0$.

(ii) If $t \leq t^{\prime}$, from the increasing property of $P(t)$, we obtain that $t \leq t_{1}$ holds if and only if $P(t) \leq P\left(t_{1}\right)=0$ and $t \geq t_{1}$ holds if and only if $P(t) \geq P\left(t_{1}\right)=0$. This completes the proof of Lemma 3.3.

From (3.4), we have the squared norm of the second fundamental form of $M^{n}$ is

$$
\begin{aligned}
S(t) & =(n-1) \lambda^{2}+\mu^{2} \\
& =\frac{n^{2}}{4} \lambda^{2}+\frac{(n-2) \beta}{4 \alpha(n-1)} \lambda+\frac{\beta^{2}}{16 \alpha^{2}(n-1)^{2}} .
\end{aligned}
$$


Putting $t=\lambda$, we have the following Lemma:

LEMMA 3.4. Let

$$
S(t)=\frac{n^{2}}{4} t^{2}+\frac{(n-2) \beta}{4 \alpha(n-1)} t+\frac{\beta^{2}}{16 \alpha^{2}(n-1)^{2}},
$$

and $t^{\prime \prime}=-\frac{(n-2) \beta}{2 \alpha n^{2}(n-1)}$. If $c+\frac{\beta^{2}}{4 \alpha^{2} n^{2}(n-1)^{2}}>0$, then

(i) If $t \geq t^{\prime \prime}$, then $t \geq t_{2}$ holds if and only if $S(t) \geq S\left(t_{2}\right)$ and $t \leq t_{2}$ holds if and only if $S(t) \leq S\left(t_{2}\right)$.

(ii) If $t \leq t^{\prime \prime}$, then $t \leq t_{1}$ holds if and only if $S(t) \geq S\left(t_{1}\right)$ and $t \geq t_{1}$ holds if and only if $S(t) \leq S\left(t_{1}\right)$.

Proof. We have

$$
\frac{d S(t)}{d t}=\frac{n^{2}}{2} t+\frac{(n-2) \beta}{4 \alpha(n-1)},
$$

it follows that the solution of $\frac{d S(t)}{d t}=0$ is $t^{\prime \prime}=-\frac{(n-2) \beta}{2 \alpha n^{2}(n-1)}$. Therefore, we know that if $t^{\prime \prime} \geq-\frac{(n-2) \beta}{2 \alpha n^{2}(n-1)}$ if and only if $S(t)$ is an increasing function, $t^{\prime \prime} \leq-\frac{(n-2) \beta}{2 \alpha n^{2}(n-1)}$ if and only if $S(t)$ is a decreasing function and $S(t)$ obtain its minimum at $t^{\prime \prime}=-\frac{(n-2) \beta}{2 \alpha n^{2}(n-1)}$.

Since $c+\frac{\beta^{2}}{4 \alpha^{2} n^{2}(n-1)^{2}}>0$, we have $P\left(t^{\prime \prime}\right)=c+\frac{(n-2) \beta^{2}}{2 \alpha^{2} n^{4}(n-1)}>0$. Thus, we have $t_{1}<$ $t^{\prime \prime}<t_{2}$.

(i) If $t \geq t^{\prime \prime}$, from the increasing property of $S(t)$, we obtain that $t \geq t_{2}$ holds if and only if $S(t) \geq S\left(t_{2}\right)$ and $t \leq t_{2}$ holds if and only if $S(t) \leq S\left(t_{2}\right)$.

(ii) If $t \leq t^{\prime \prime}$, from the decreasing property of $S(t)$, we obtain that $t \leq t_{1}$ holds if and only if $S(t) \geq S\left(t_{1}\right)$ and $t \geq t_{1}$ holds if and only if $S(t) \leq S\left(t_{1}\right)$. This completes the proof of Lemma 3.4 .

Proof of Main Theorem 1.2. Putting $t=\lambda$, from (3.21), we have

$$
\frac{d^{2} \varpi}{d s^{2}}+\varpi P(t)=0 .
$$

(1) If $\min \left(S\left(t_{1}\right), S\left(t_{2}\right)\right) \leq S(t) \leq \max \left(S\left(t_{1}\right), S\left(t_{2}\right)\right)$, then we have $S\left(t_{1}\right) \leq S(t) \leq$ $S\left(t_{2}\right)$ or $S\left(t_{2}\right) \leq S(t) \leq S\left(t_{1}\right)$.

(i) If $S\left(t_{1}\right) \leq S(t) \leq S\left(t_{2}\right)$, we consider two cases $t \geq t^{\prime \prime}$ or $t<t^{\prime \prime}$.

Case $(i)$. If $t \geq t^{\prime \prime}$, we also consider two subcases $t^{\prime \prime} \geq t^{\prime}$ or $t^{\prime \prime}<t^{\prime}$.

Subcase ( $i)$. If $t^{\prime \prime} \geq t^{\prime}$, we have $t \geq t^{\prime}$. Since $S(t) \leq S\left(t_{2}\right)$, from Lemma 3.4, Lemma 3.3 and (3.31), we have $S(t) \leq S\left(t_{2}\right)$ holds if and only if $t \leq t_{2}$ if and only if $P(t) \geq 0$ and if and only if $\frac{d^{2} \varpi}{d s^{2}} \leq 0$. Thus $\frac{d \varpi}{d s}$ is a monotonic function of $s \in(-\infty,+\infty)$. Therefore, by the similar assertion in Wei [11], we have $\varpi(s)$ must be monotonic when $s$ tends to infinity. From Lemma 3.2, we have the positive function $\varpi(s)$ is bounded. By the same assertion in the proof of Main Theorem 1.1, we know that $M^{n}$ is isometric to the Riemannin product $R^{1} \times S^{n-1}(a)$ or $R^{n-1} \times S^{1}(a)$ for $c=0$ and $\beta \neq 0$; or $S^{1}(a) \times S^{n-1}\left(\sqrt{1-a^{2}}\right)$ for $c=1$; or $H^{1}\left(\tanh ^{2} \rho-1\right) \times S^{n-1}\left(\operatorname{coth}^{2} \rho-1\right)$ or $H^{n-1}\left(\tanh ^{2} \rho-1\right) \times S^{1}\left(\operatorname{coth}^{2} \rho-1\right)$ for $c=-1$ and $\beta^{2}-4 \alpha^{2} n^{2}(n-1)^{2}>0$. 
Subcase (ii). If $t^{\prime \prime}<t^{\prime}$, since $t \geq t^{\prime \prime}$, we have $t^{\prime \prime} \leq t<t^{\prime}$ or $t \geq t^{\prime}$.

If $t^{\prime \prime} \leq t<t^{\prime}$, from the increasing property of $P(t)$, we have $P(t) \geq P\left(t^{\prime \prime}\right)>0$. From (3.31), we have $\frac{d^{2} \varpi}{d s^{2}}<0$. This implies that $\frac{d \omega(s)}{d s}$ is a strictly monotone decreasing function of $s$ and thus it has at most one zero point for $s \in(-\infty,+\infty)$. If $\frac{d \varpi(s)}{d s}$ has no zero point in $(-\infty,+\infty)$, then $\varpi(s)$ is a monotone function of $s$ in $(-\infty,+\infty)$. If $\frac{d \varpi(s)}{d s}$ has exactly one zero point $s_{0}$ in $(-\infty,+\infty)$, then $\varpi(s)$ is a monotone function of $s$ in both $\left(-\infty, s_{0}\right]$ and $\left[s_{0},+\infty\right)$.

On the other hand, from Lemma 3.2, we know that $\varpi(s)$ is bounded. Since $\varpi(s)$ is bounded and monotonic when $s$ tends to infinity, we know that both $\lim _{s \rightarrow-\infty} \varpi(s)$ and $\lim _{s \rightarrow+\infty} \varpi(s)$ exist and (3.27) holds. This is impossible because $\frac{d \varpi(s)}{d s}$ is a strictly monotone decreasing function of $s$. Therefore, we know that the case $t^{\prime \prime} \leq t<t^{\prime}$ does not occur and we conclude that $t \geq t^{\prime}$.

If $t \geq t^{\prime}$, then $t>t^{\prime \prime}$. Since $S(t) \leq S\left(t_{2}\right)$, from Lemma 3.4, Lemma 3.3 and (3.31), we have $S(t) \leq S\left(t_{2}\right)$ holds if and only if $t \leq t_{2}$ if and only if $P(t) \geq 0$ and if and only if $\frac{d^{2} \varpi}{d s^{2}} \leq 0$. Thus $\frac{d \varpi}{d s}$ is a monotonic function of $s \in(-\infty,+\infty)$. By the same assertion in the proof of Main Theorem 1.1, we know that (1) in Main Theorem 1.2 is true.

Case (ii). If $t<t^{\prime \prime}$, we also consider two subcases $t^{\prime \prime} \geq t^{\prime}$ or $t^{\prime \prime}<t^{\prime}$.

Subcase (i). If $t^{\prime \prime} \geq t^{\prime}$, since $t<t^{\prime \prime}$, we have $t^{\prime}<t<t^{\prime \prime}$ or $t \leq t^{\prime}$.

If $t^{\prime}<t<t^{\prime \prime}$, from the decreasing property of $P(t)$, we have $P(t)>P\left(t^{\prime \prime}\right)>0$. From (3.31), we have $\frac{d^{2} \varpi}{d s^{2}}<0$. This implies that $\frac{d \varpi(s)}{d s}$ is a strictly monotone decreasing function of $s$ and thus it has at most one zero point for $s \in(-\infty,+\infty)$. By the same assertion in the proof of Case (i), we know that the case $t^{\prime}<t<t^{\prime \prime}$ does not occur and we conclude that $t \leq t^{\prime}$.

If $t \leq t^{\prime}$, since $t<t^{\prime \prime}$ and $S(t) \geq S\left(t_{1}\right)$, from Lemma 3.4, Lemma 3.3 and (3.31), we have $S(t) \geq S\left(t_{1}\right)$ holds if and only if $t \leq t_{1}$ if and only if $P(t) \leq 0$ and if and only if $\frac{d^{2} \varpi}{d s^{2}} \geq 0$. Thus $\frac{d \varpi}{d s}$ is a monotonic function of $s \in(-\infty,+\infty)$. By the same assertion in the proof of Main Theorem 1.1, we know that (1) in Main Theorem 1.2 is true.

Subcase (ii). If $t^{\prime \prime}<t^{\prime}$, since $t<t^{\prime \prime}$, we have $t<t^{\prime}$. Since $S(t) \geq S\left(t_{1}\right)$, from Lemma 3.4, Lemma 3.3 and (3.31), we have $S(t) \geq S\left(t_{1}\right)$ holds if and only if $t \leq t_{1}$ if and only if $P(t) \leq 0$ and if and only if $\frac{d^{2} \varpi}{d s^{2}} \geq 0$. Thus $\frac{d \omega}{d s}$ is a monotonic function of $s \in(-\infty,+\infty)$. By the same assertion in the proof of Main Theorem 1.1, we know that (1) in Main Theorem 1.2 is true.

(ii) If $S\left(t_{2}\right) \leq S(t) \leq S\left(t_{1}\right)$, we also consider two cases $t \geq t^{\prime \prime}$ or $t<t^{\prime \prime}$. By the same assertion in the proof of (i), we know that (1) in Main Theorem 1.2 is true.

(2) If $S(t) \geq \max \left(S\left(t_{1}\right), S\left(t_{2}\right)\right)$, we consider two cases $t \geq t^{\prime \prime}$ or $t<t^{\prime \prime}$.

Case $(i)$. If $t \geq t^{\prime \prime}$, we also consider two subcases $t^{\prime \prime} \geq t^{\prime}$ or $t^{\prime \prime}<t^{\prime}$.

Subcase (i). If $t^{\prime \prime} \geq t^{\prime}$, we have $t \geq t^{\prime}$. Since $S(t) \geq \max \left(S\left(t_{1}\right), S\left(t_{2}\right)\right)$, we have $S(t) \geq S\left(t_{2}\right)$, from Lemma 3.4, Lemma 3.3 and (3.31), we have $S(t) \geq S\left(t_{2}\right)$ holds if and only if $t \geq t_{2}$ if and only if $P(t) \leq 0$ and if and only if $\frac{d^{2} \varpi}{d s^{2}} \geq 0$. Thus $\frac{d \varpi}{d s}$ is a monotonic function of $s \in(-\infty,+\infty)$. By the same assertion in the proof of Main Theorem 1.1, we know that (2) in Main Theorem 1.2 is true. 
Subcase (ii). If $t^{\prime \prime}<t^{\prime}$, since $t \geq t^{\prime \prime}$, we have $t^{\prime \prime} \leq t<t^{\prime}$ or $t \geq t^{\prime}$.

If $t^{\prime \prime} \leq t<t^{\prime}$, from the increasing property of $P(t)$, we have $P(t) \geq P\left(t^{\prime \prime}\right)>0$. From (3.31), we have $\frac{d^{2} \varpi}{d s^{2}}<0$. This implies that $\frac{d \varpi(s)}{d s}$ is a strictly monotone decreasing function of $s$. By the same assertion in the proof of Case (i) in (1), we know that the case $t^{\prime \prime} \leq t<t^{\prime}$ does not occur and we conclude that $t \geq t^{\prime}$.

If $t \geq t^{\prime}$, then $t>t^{\prime \prime}$. Since $S(t) \geq S\left(t_{2}\right)$, from Lemma 3.4, Lemma 3.3 and (3.31), we have $\frac{d^{2} w}{d s^{2}} \geq 0$. By the same assertion above, we know that (2) in Main Theorem 1.2 is true.

Case (ii). If $t<t^{\prime \prime}$, we also consider two subcases $t^{\prime \prime} \geq t^{\prime}$ or $t^{\prime \prime}<t^{\prime}$.

Subcase (i). If $t^{\prime \prime} \geq t^{\prime}$, since $t<t^{\prime \prime}$, we have $t^{\prime}<t<t^{\prime \prime}$ or $t \leq t^{\prime}$.

If $t^{\prime}<t<t^{\prime \prime}$, from the decreasing property of $P(t)$, we have $P(t)>P\left(t^{\prime \prime}\right)>0$. From (3.31), we have $\frac{d^{2} \varpi}{d s^{2}}<0$. This implies that $\frac{d \varpi(s)}{d s}$ is a strictly monotone decreasing function of $s$. By the same assertion in the proof of Case (i) in (1), we know that the case $t^{\prime}<t<t^{\prime \prime}$ does not occur and we conclude that $t \leq t^{\prime}$.

If $t \leq t^{\prime}$, since $t<t^{\prime \prime}$ and $S(t) \geq \max \left(S\left(t_{1}\right), S\left(t_{2}\right)\right)$, we have $S(t) \geq S\left(t_{1}\right)$, from Lemma 3.4, Lemma 3.3 and (3.31), we have $S(t) \geq S\left(t_{1}\right)$ holds if and only if $t \leq t_{1}$ if and only if $P(t) \leq 0$ and if and only if $\frac{d^{2} \varpi}{d s^{2}} \geq 0$. Thus $\frac{d \sigma}{d s}$ is a monotonic function of $s \in(-\infty,+\infty)$. By the same assertion in the proof of Main Theorem 1.1, we know that (2) in Main Theorem 1.2 is true.

Subcase (ii). If $t^{\prime \prime}<t^{\prime}$, since $t<t^{\prime \prime}$, we have $t<t^{\prime}$. Since $S(t) \geq S\left(t_{1}\right)$, from Lemma 3.4, Lemma 3.3 and (3.31), we have $\frac{d^{2} \varpi}{d s^{2}} \geq 0$. By the same assertion above, we know that (2) in Main Theorem 1.2 is true.

(3) If $S(t) \leq \min \left(S\left(t_{1}\right), S\left(t_{2}\right)\right)$, we consider two cases $t \geq t^{\prime \prime}$ or $t<t^{\prime \prime}$.

Case (i). If $t \geq t^{\prime \prime}$, we also consider two subcases $t^{\prime \prime} \geq t^{\prime}$ or $t^{\prime \prime}<t^{\prime}$.

Subcase (i). If $t^{\prime \prime} \geq t^{\prime}$, we have $t \geq t^{\prime}$. Since $S(t) \leq \min \left(S\left(t_{1}\right), S\left(t_{2}\right)\right)$, we have $S(t) \leq S\left(t_{2}\right)$, from Lemma 3.4, Lemma 3.3 and (3.31), we have $S(t) \leq S\left(t_{2}\right)$ holds if and only if $t \leq t_{2}$ if and only if $P(t) \geq 0$ and if and only if $\frac{d^{2} \varpi}{d s^{2}} \leq 0$. Thus $\frac{d \omega}{d s}$ is a monotonic function of $s \in(-\infty,+\infty)$. By the same assertion in the proof of Main Theorem 1.1, we know that (3) in Main Theorem 1.2 is true.

Subcase (ii). If $t^{\prime \prime}<t^{\prime}$, since $t \geq t^{\prime \prime}$, we have $t^{\prime \prime} \leq t<t^{\prime}$ or $t \geq t^{\prime}$.

If $t^{\prime \prime} \leq t<t^{\prime}$, from the increasing property of $P(t)$, we have $P(t) \geq P\left(t^{\prime \prime}\right)>0$. From (3.31), we have $\frac{d^{2} w}{d s^{2}}<0$. By the same assertion in the proof of Case (i) in (1), we know that the case $t^{\prime \prime} \leq t<t^{\prime}$ does not occur and we conclude that $t \geq t^{\prime}$.

If $t \geq t^{\prime}$, then $t>t^{\prime \prime}$. Since $S(t) \leq S\left(t_{2}\right)$, from Lemma 3.4, Lemma 3.3 and (3.31), we have $\frac{\bar{d}^{2} \omega}{d s^{2}} \leq 0$. By the same assertion above, we also know that (3) in Main Theorem 1.2 is true.

Case (ii). If $t<t^{\prime \prime}$, we also consider two subcases $t^{\prime \prime} \geq t^{\prime}$ or $t^{\prime \prime}<t^{\prime}$.

Subcase (i). If $t^{\prime \prime} \geq t^{\prime}$, since $t<t^{\prime \prime}$, we have $t^{\prime}<t<t^{\prime \prime}$ or $t \leq t^{\prime}$.

If $t^{\prime}<t<t^{\prime \prime}$, from the decreasing property of $P(t)$, we have $P(t)>P\left(t^{\prime \prime}\right)>0$. From (3.31), we have $\frac{d^{2} \varpi}{d s^{2}}<0$. By the same assertion in the proof of Case (i) 
in (1), we know that the case $t^{\prime}<t<t^{\prime \prime}$ does not occur and we conclude that $t \leq t^{\prime}$.

If $t \leq t^{\prime}$, since $t<t^{\prime \prime}$ and $S(t) \leq \min \left(S\left(t_{1}\right), S\left(t_{2}\right)\right)$, we have $S(t) \leq S\left(t_{1}\right)$, from Lemmas 3.3 and 3.4 and (3.31), we have $S(t) \leq S\left(t_{1}\right)$ holds if and only if $t \geq t_{1}$ if and only if $P(t) \geq 0$ and if and only if $\frac{d^{2} \varpi}{d s^{2}} \leq 0$. Thus $\frac{d \varpi}{d s}$ is a monotonic function of $s \in(-\infty,+\infty)$. By the same assertion in the proof of Main Theorem 1.1, we know that (3) in Main Theorem 1.2 is true.

Subcase (ii). If $t^{\prime \prime}<t^{\prime}$, since $t<t^{\prime \prime}$, we have $t<t^{\prime}$. Since $S(t) \leq S\left(t_{1}\right)$, from Lemma 3.4, Lemma 3.3 and (3.31), we have $\frac{d^{2} \varpi}{d s^{2}} \leq 0$. By the same assertion above, we know that (3) in Main Theorem 1.2 is true. This completes the proof of Main Theorem 1.2.

ACKNOWLEDGEMENTS. This project was supported by NSF of Shaanxi Province(SJ08A31)and NSF of Shaanxi Educational Committee (2008JK484).

\section{REFERENCES}

1. E. Cartan, Sur des familles remarquables d'hypersurfaces isoparamétriques dans les espaces sphériques, Math. Z. 45 (1939), 335-367.

2. S. Y. Cheng and S. T. Yau, Hypersurfaces with constant scalar curvature, Math. Ann. 225 (1977), 195-204.

3. Q. M. Cheng, Hypersurfaces in a unit sphere $S^{n+1}(1)$ with constant scalar curvature, J. Lond. Math. Soc. 64 (2001), 755-768.

4. Q. M. Cheng, Complete hypersurfaces in a Euclidean space $R^{n+1}$ with constant scalar curvature, Indiana Univ. Math. J. 51 (2002), 53-68.

5. Z. Hu and S. Zhai, Hypersurfaces of the hyperbolic space with constant scalar curvature, Results Math. 48 (2005), 65-88. 351.

6. H. Li. Global rigidity theorems of hypersurfaces, Ark. Mat. 35 (1997), 327-

7. H. Li, Y. J. Suh and G. Wei, Linear Weingarten hypersurfaces in a unit sphere, Bull. Korean Math. Soc. 46 (2009), 321-329.

8. T. Otsuki, Minimal hypersurfaces in a Riemannian manifold of constant curvature, Amer. J. Math. 92 (1970), 145-173.

9. S. Shu and A. Yi Han, Hypersurfaces in a hyperbolic space with constant $k$-th mean curvature, Bull. Math. Soc. Sci. Math. Roumanie 52(100) (2009), 65 C78.

10. S. Shu and A. Yi Han, Nonnegative sectional curvature hypersurfaces in a real space form, Math. Notes 86 (2009), 729-743.

11. G. Wei, Complete hypersurfaces with constant mean curvature in a unit sphere, Monatsh. Math. 149 (2006), 251-258. 